\title{
True negative fraction
}

Margaret Pepe

\section{Source}

Margaret Sullivan Pepe. (2003). The Statistical Evaluation of Medical Tests for

Classification and Prediction Oxford University Press

The fraction (percentage) of subjects without a given disease correctly identified as such. Also known as true negative rate or specificity. 